\title{
La formación de los emprendedores y sus consecuencias sobre la innovación y el éxito empresarial '
}

\author{
Training of entrepreneurs and their consequences on innovation \\ and business success \\ ' Natalia Martín Cruz, Juan Hernangómez Barahona, Ana Isabel Rodríguez Escudero \\ y ${ }^{2}$ Fernando Saboia Leitao \\ ' Departamento de Organización de Empresas y Comercialización e Investigación de Mercados. \\ Universidad de Valladolid. Avda. Valle Esgueva, n 6, 4701 I - Valladolid, \\ ${ }^{2}$ Rua Dom Sebastião Leme, 255 / I02-B CEP: 60050-I 60 Fortaleza/CE Brasil. \\ Universidad Federal de Ceará. \\ ambiela@eco.uva.es jhernan@eco.uva.es ana@eco.uva.es saboia@ufc.br
}

Fecha de recepción: 10-03-2010

Fecha de aceptación: 18-06-2010

Resumen: Utilizando los razonamientos de la teoría de la conducta planificada, este trabajo pretende arrojar luz sobre el efecto de los programas de formación del emprendedor en la innovación y el éxito empresarial. De manera complementaria, se evalúa el impacto sobre estas dos variables de la personalidad y la experiencia del individuo. Para cumplir con los objetivos planteados utilizamos una muestra de 354 emprendedores de Castilla y León. Los resultados obtenidos nos llevan a recomendar a los centros de formación, e instituciones que los gestionan, que den preferencia a la formación específica emprendedora -y no tanto a la formación generalista en aspectos empresariales-, cuando se quiere reforzar la innovación.

Palabras clave: Formación de los emprendedores, personalidad, experiencia laboral, innovación, éxito empresarial.

Abstract: We use the planned behavioural theory to valuate the influence of training programs on innovation and business success. Additionally, we measure the effect of personality and experience of the individual on innovation and business success. In this paper, we propose to study what type of training is most appropriate in order to reinforce such a capacity. To achieve this objective, we used a sample of 354 entrepreneurs from Castile and Leon. The results obtained lead us to recommend that the training centres and the institutions which manage them should give preference to specific entrepreneurial training -and not as much to general training in business aspects- when they wish to strengthen the innovating spirit of the entrepreneurs.

Key words: Entrepreneurial training, personality, job experience, innovation, business success.

\section{La formación de los emprendedores y la teoría de la conducta planificada}

Los programas de formación de los emprendedores (PFE) tienen como fin último influir en el comportamiento emprendedor futuro de los individuos y en el éxito de este comportamiento. Desde que en 1947 se pusiera en marcha el primer PFE en la Universidad de Harvard en Estados Unidos (Katz, 2003), este tipo de programas ha tenido un gran desarroIlo, tanto en este país como en el resto del mundo. Tal como reconocen Béchard y Grégoire (2005) se han ido sucediendo trabajos de investigación que se centran en diversos aspectos de este tipo de educación. Por ejemplo, ha sido ampliamente estudiada la relación entre las características de los centros formativos y sus programas de formación o la relación entre los programas y el perfil de los participantes.

En todo caso, una característica de este tipo de estos estudios es que la mayoría versan sobre la enseñanza universitaria (Gorman et al., 1997), no sobre la que se imparte en otro tipo de instituciones (Cámaras de Comercio, Confederaciones de Empresarios, etc.). Sin

\footnotetext{
I Para la realización de este trabajo hemos contado con la subvención de la Consejería de Educación y Ciencia de la Junta de Castilla y León (proyecto BU00IA08), a la que manifestamos nuestro agradecimiento.
} 
embargo, muchas instituciones públicas promueven PFE por la importancia que el comportamiento emprendedor tiene sobre el desarrollo económico y la competitividad del tejido empresarial (Hytti y Kuopusjarvi, 2004). Por esta razón, este trabajo pretende arrojar luz sobre los efectos de los programas formativos orientados al emprendedor teniendo en cuenta la diversidad de la oferta.

En nuestro estudio nos apoyamos en el marco teórico derivado de los planteamientos de la Teoría de la Conducta Planificada (TCP) para analizar el impacto de los PFE en la conducta emprendedora. La TCP ha sido utilizada en numerosos contextos, áreas y poblaciones para evaluar intenciones y comportamientos individuales (Ajzen, 2008; Ajzen y Manstead, 2007) y, recientemente, se ha empezado a aplicar al contexto de los PFE (Fayolle et al., 2006; Souitaris et al., 2007; Florin et al., 2007). En concreto, Fayolle et al. (2006) exponen cómo los PFE influyen sobre los tres factores recogidos en la TCP, tal como se representa en la Figura I. Más específicamente, revelan como determinados cambios en la intención y, en última instan- cia, en la conducta emprendedora y en los resultados de esa conducta, son consecuencia de la influencia de los PFE en los antecedentes de la TCP. Concretamente, según esta teoría aplicada al ámbito de los PFE, la formación generará autoconfianza en la propia capacidad (Krueger y Carsrud, 1993), fortalecerá el conocimiento de las normas sociales y de los factores del entorno ( $y$, por tanto, la creencia de que se pueden controlar) y, en definitiva, reforzará la conducta emprendedora y mejorará sus resultados.

La aproximación que utilizamos a la conducta emprendedora es el comportamiento innovador. Nos centramos en este aspecto ya que, siguiendo a Schumpeter, constituye su verdadera esencia. Es decir, un emprendedor es aquella persona que rompe las convenciones y crea nuevos productos, nuevas formas de producir, nuevos modelos de negocio (Mayer-Schönberger, 2007).

Más allá del efecto central que planteamos sobre la influencia de los PFE en la innovación, se utilizan una serie de relaciones de control (Figura 2) para des-

Figura I

Los programas de formación de los emprendedores (PFE) y la teoría de la conducta planificada (TCP)

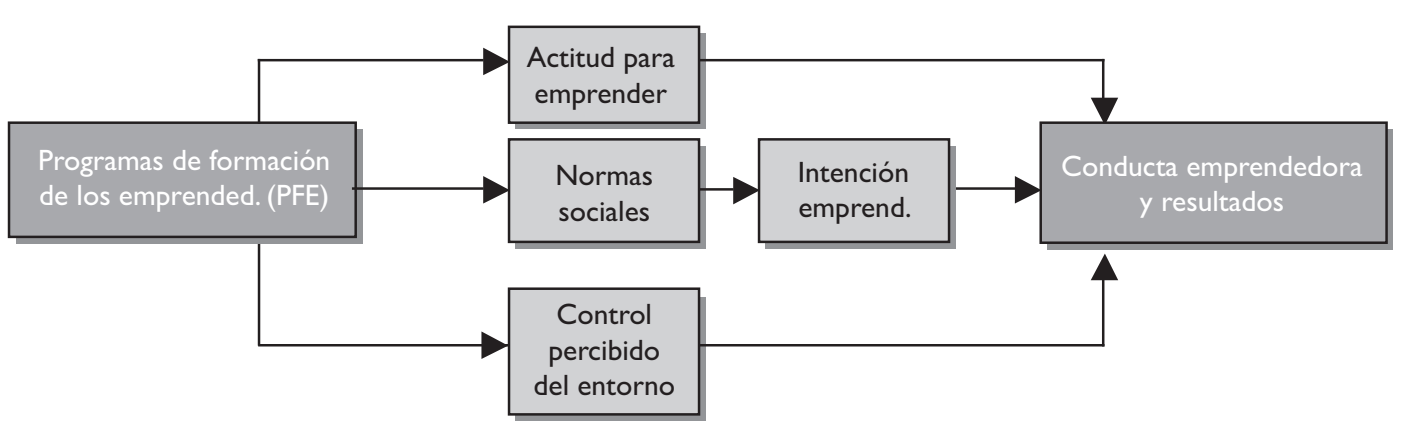

Figura 2

Modelo planteado

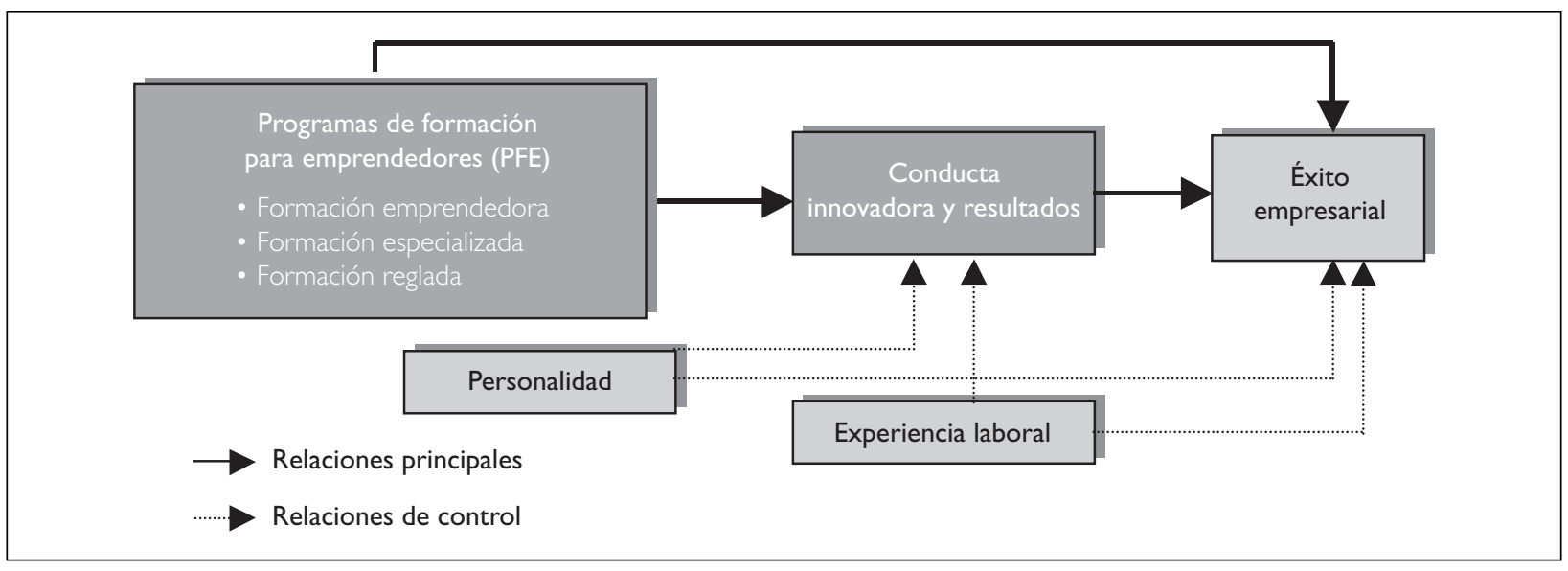


cartar que los efectos encontrados pudieran tener su explicación en variables no contempladas. Concretamente, se incluye la personalidad del emprendedor (Cross y Travaglione, 2003; lakovleva y Kolvereid, 2005; Hisrich et al., 2005) y su experiencia laboral (Burke et al., 2000; Capellas et al., 2004; Wandosell y Garcia, 2004) como variables con potencial para influir en la decisión y en el resultado de innovar por parte de un individuo, variables que, a su vez, pueden estar relacionadas con el nivel de formación del individuo. Además, el trabajo incluye el contraste de la influencia tradicionalmente atribuida a la innovación sobre el éxito; la literatura previa avala esta relación (Utsch y Rauch, 2000; Avlonitis y Salavou, 2007; Comacchio et al., 2007; Yong y Weng Ho, 2006).

\section{Metodología}

\section{I. Unidad de análisis y muestra}

Para dar cumplimiento a los objetivos planteados utilizamos una muestra de 354 emprendedores extraída de la base de datos de 30.000 emprendedores de CECALE. Las unidades muestrales analizadas pertenecen a las nueve provincias de la comunidad de Castilla y León, provincias representadas de forma proporcional a su número de emprendedores. El concepto de emprendedor que se utiliza en la presente investigación es el de aquel individuo que posee simultáneamente la condición de propietario y directivo de un negocio y que tiene 5 ó más empleados (Djankov et al., 2005). Se contempla esta última característica con el propósito de no incluir en la muestra a los denominados autónomos.

La caracterización más básica de los emprendedores de la muestra nos lleva a considerar dos rasgos socio-demográficos: la edad y el sexo. La mayoría de los emprendedores son hombres, un $84 \%$, frente a un $16 \%$ de mujeres. Por su parte, la edad más frecuente de los emprendedores es de 42 años y el intervalo en el que se agrupa un $40 \%$ de la muestra es el de 36 a 45 años, aunque nos encontramos tanto con individuos muy jóvenes ( 19 años) como con emprendedores que han sobrepasado la edad normal de jubilación (70 años). Asimismo, contamos con representantes de las cuatro grandes áreas de actividad: servicios, comercio, agricultura e industria. La participación mayoritaria corresponde al sector servicios (43\%), seguido por la industria (29\%) y el comercio (24\%). En último lugar, y bastante distanciado, se encuentra el sector agrícola (4\%) (Cuadro I de la página siguiente).
En cuanto al año de constitución y, por tanto, la antigüedad de las empresas estudiadas, la mayoría se crean a partir de la década de los noventa (concretamente, más del $50 \%$ a partir de 1992). Además, el $25 \%$ del total de emprendedores fundó su negocio a partir de 1997. En todo caso, también están recogidos en la muestra emprendedores que dirigen una empresa familiar constituida hace más de cien años (la más antigua es de 1898). Por fin, en cuanto al porcentaje del capital que tienen los emprendedores analizados, la mayoría disfruta del $50 \%$ o más, con lo que se puede afirmar que son quiénes dirigen el futuro y toman decisiones estratégicas. La cifra de ventas, expresada en miles de euros, es muy heterogénea ya que no hay ninguno de los seis intervalos cuenta con más del $20 \%$ de la población de emprendedores analizados (Cuadro I).

Como resumen de todo ello podemos caracterizar el perfil del empresario de la muestra como el de un hombre, que ha puesto en marcha un proyecto empresarial en el sector servicios, comercio o industria durante los últimos 20 años, que controla la mayoría del capital y que tiene un número de empleados que no supera los 50.

\subsection{Medición de las variables}

La personalidad del individuo se ha medido con la escala de 12 ítemes que se puede ver en el Cuadro 2. El encuestado ha valorado las proposiciones planteadas en un rango de I a 5, donde I significa «nunca» y 5 «siempre». Para la construcción de esta escala nos basamos en trabajos previos en los que se ha medido la personalidad del emprendedor (Mescon y Montanari, 1981; Baum et al., 200 I; Baron, 2004; Howell, 2005). Por su parte, la experiencia se ha determinado a partir del número de años trabajados en las áreas de marketing, finanzas y recursos humanos. La escala ordinal de medición utilizada ha sido la siguiente: 0 «ninguna experiencia», I «menos de dos años», 2 «de dos a cinco años», 3 «de cinco a ocho años», 4 «más de ocho años». El valor medio de los ítemes de esta escala está en torno al 2,5 y la mediana es 3 .

Haciéndonos eco del debate sobre los métodos alternativos para capacitar al emprendedor (Honing, 2004), la formación se ha valorado en tres niveles: formación emprendedora propiamente dicha, formación especializada en aspectos empresariales y formación reglada. En el primer nivel, se ha considerado tanto la realización de cursos en instituciones al efecto, como con la preparación obtenida de for- 
Cuadro I

Características básicas de los emprendedores y de los proyectos

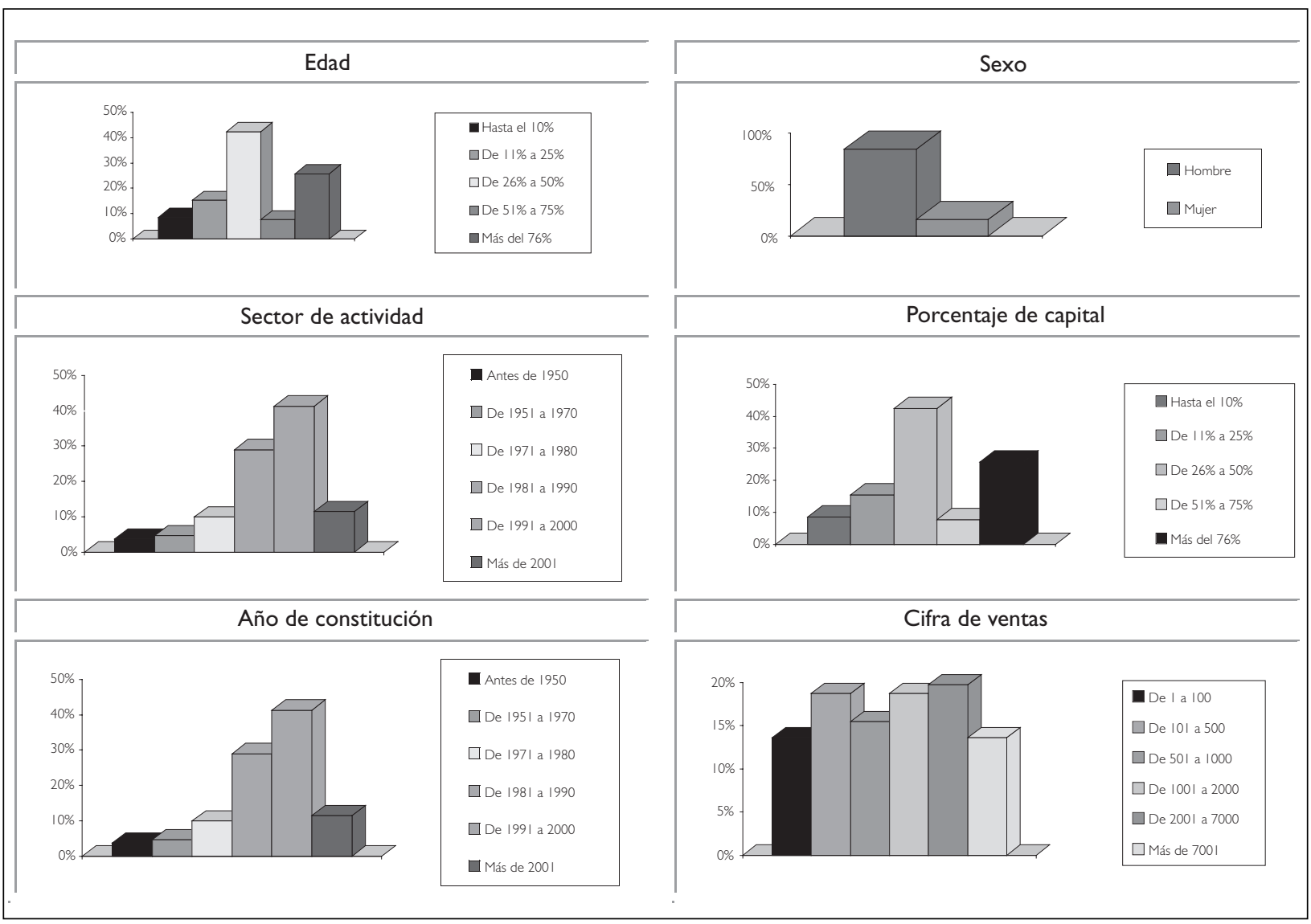

ma autodidacta, en perfeccionamiento directivo y capacitación emprendedora. En el segundo nivel, que también recoge la formación en instituciones y autodidacta, se ha medido la especialización en áreas de la empresa (marketing, finanzas, etc.) y en temas relacionados con la actividad de la empresa. Para los ocho ítemes que forman estos constructos relativos a la formación (véase el Cuadro 2), la escala de medición ha sido la siguiente: 0 «nunca», I «menos de 30 horas», 2 «entre 30 y 200 horas», 3 «entre 200 y 1200 horas», 4 «más de $\mid 200$ horas».

Tal como se desprende de las medias presentadas en el Cuadro 2, en términos generales podemos decir que los emprendedores no han seguido de forma masiva cursos de formación emprendedora. De hecho, la moda para los cuatro ítemes de esta escala es 0 y la mediana de I. Los cursos de capacitación emprendedora son los que menos interés han despertado, ya que únicamente el $43 \%$ de los encuestados han seguido algún curso relacionado específicamente con las características del emprendedor y, cuando lo han hecho, generalmente han sido cursos de menos de 30 horas (el 16,2\%) o de entre 30 y 200 ho- ras (un 18, 1\%). El perfeccionamiento directivo ha despertado un poco más de interés; el $65 \%$ de los emprendedores han seguido cursos orientados a mejorar sus cualidades directivas. Concretamente, de entre 30 y 200 horas, el 25,9\% de los emprendedores, y de 200 a 1200 horas, el 18,1\% de los emprendedores. La formación autodidacta en estas materias sigue una tendencia similar a la formación en centros.

La formación especializada en áreas de la empresa ha contado con un mayor seguimiento. Una de las medias de los ítemes de esta variable está por encima de 2 y otro muy próximo. A su vez, los valores de las medianas son 2. El análisis de frecuencia indica que un 70,2\% ha realizado, en algún momento de su vida, cursos de especialización funcional, que mayoritariamente han tenido una duración de entre 30 y 200 horas. Finalmente, los cursos de especialización en la actividad de la empresa son los que más han interesado a los emprendedores (el 79,4\%). La preferencia de duración de este tipo de cursos también ha sido de entre 30 y 200 horas, aunque un 23,9\% de los emprendedores ha realizado cursos de entre 
200 y 1200 horas. De nuevo la formación autodidacta manifiesta una misma pauta que la formación en centros.

Para determinar la formación reglada se han utilizado los niveles habituales: I «EGB / ESO», 2 «BUPCOU / Bachillerato / FP» 3 «Formación Profesional Superior / Diplomatura», 4 «Licenciatura / Ingeniería», 5 «MBA / Doctorado». La distribución de los emprendedores por su nivel de formación está bastante repartida en los diferentes niveles que ofrece el sistema educativo español. Un gran porcentaje de los emprendedores $(28,8 \%)$ ha realizado una formación profesional superior o una diplomatura; en segundo lugar, aparecen los emprendedores que han llegado a cursar el bachillerato o una formación profesional de primer ciclo (27,6\%). Los licenciados o ingenieros componen el tercer grupo de emprendedores en porcentaje $(21,2 \%)$. Los que sólo concluyeron la edu- cación primaria suponen el I4,5\% del total; $y$, por fin, el 7,9\% tiene un MBA o doctorado.

Finalmente, la conducta innovadora se ha medido preguntando por el «grado de satisfacción con la innovación en productos y servicios» y el éxito empresarial por el «grado de satisfacción con la rentabilidad económica», en línea con investigaciones previas en las que se pregunta a los directivos y emprendedores acerca de su percepción sobre la innovación (Schulze y Hoegl, 2008). Para ambas variables la escala ha sido la misma: I «muy baja» a 5 «muy alta». Una media de 3,66 y una mediana y moda de 4 (que se corresponde con «alto» en la escala) para la primera de las variables, revelan el importante nivel de satisfacción que muestran los emprendedores de la muestra con su actividad innovadora. De la misma forma, la media de 3,0 I y mediana y moda de 3 para la rentabilidad económica sitúan en un nivel

Cuadro 2

Escalas de medición y estadísticos descriptivos

\begin{tabular}{|c|c|c|c|c|c|}
\hline PERSONALIDAD & Media & D.S. & FORMACIÓN EMPRENDEDORA & Media & D.S. \\
\hline Tiene facilidad para generar nuevas ideas. & 3,75 & 1,05 & $\begin{array}{l}\text { Cursos de perfeccionamiento directivo en } \\
\text { centros. }\end{array}$ & ।,4 & 1,26 \\
\hline $\begin{array}{l}\text { Tiene facilidad para empezar nuevas } \\
\text { actividades. }\end{array}$ & 3,69 & 1,10 & $\begin{array}{l}\text { Cursos de capacitación emprendedora en } \\
\text { centros. }\end{array}$ & 0,80 & I,05 \\
\hline $\begin{array}{l}\text { Identifica oportunidades donde nadie las } \\
\text { observa. }\end{array}$ & 3,02 & 1,13 & Perfeccionamiento directivo autodidacta. & ।,37 & 1,20 \\
\hline $\begin{array}{l}\text { Si decide hacer algo, lo hará y nadie podrá } \\
\text { detenerle. }\end{array}$ & 3,41 & 1,13 & Capacitación emprendedora autodidacta. & 1,06 & 1,13 \\
\hline Le gusta intercambiar opiniones e ideas con & 4,15 & 0,88 & FORMACIÓN ESPECIALIZADA & Media & D.S. \\
\hline $\begin{array}{l}\text { otras personas. } \\
\text { Tiene facilidad para relacionarse con la }\end{array}$ & 4.15 & 0,88 & $\begin{array}{l}\text { Cursos de especialización (Marketing, } \\
\text { Finanzas, RR.HH, otros) en centros. }\end{array}$ & ।,48 & $|, 2|$ \\
\hline $\begin{array}{l}\text { gente. } \\
\text { Procura que sus decisiones tengan }\end{array}$ & 4,36 & 0,76 & $\begin{array}{l}\text { Cursos de especialización en temas de la } \\
\text { actividad de la empresa en centros. }\end{array}$ & ।,99 & 1,34 \\
\hline $\begin{array}{l}\text { Procura cumplir con los compromisos que } \\
\text { asume. }\end{array}$ & 4,61 & 0,67 & $\begin{array}{l}\text { Especialización (Marketing, Finanzas, RR.HH, } \\
\text { otros) autodidacta. }\end{array}$ & ।,43 & 1,18 \\
\hline $\begin{array}{l}\text { Le gusta disfrutar y ser reconocido por sus } \\
\text { éxitos personales. }\end{array}$ & 3,40 & 1,17 & $\begin{array}{l}\text { Especialización en temas de la actividad de } \\
\text { la empresa autodidacta. }\end{array}$ & 2,11 & 1,28 \\
\hline Cree que ser emprendedor de éxito tiene & 3,24 & 1,19 & FORMACIÓN REGLADA & Media & D.S. \\
\hline reconocimiento social. & & & Nivel de formación reglada. & 2,80 & 1,16 \\
\hline $\begin{array}{l}\text { En los actos públicos y sociales, tiene } \\
\text { cuidado con los aspectos de protocolo. }\end{array}$ & 3,43 & 1,15 & CONDUCTA INNOVADORA & Media & D.S. \\
\hline Le gusta preservar y mantener su reputación. & 4,10 & 0,97 & $\begin{array}{l}\text { Satisfacción con el nivel de innovación en } \\
\text { productos y servicios. }\end{array}$ & 3,66 & 0,92 \\
\hline EXPERIENCIA & Media & D.S. & ÉXITO EMPRESARIAL & Media & D.S. \\
\hline $\begin{array}{l}\text { Experiencia laboral en finanzas. } \\
\text { Experiencia laboral en marketing. }\end{array}$ & $\begin{array}{l}2,63 \\
2,39\end{array}$ & $\begin{array}{l}1,57 \\
1,65\end{array}$ & Satisfacción con la rentabilidad económica. & 3,01 & 0,97 \\
\hline Experiencia laboral en recursos humanos. & 2,45 & 1,59 & & & \\
\hline
\end{tabular}


«medio» el grado de apreciación del éxito de su empresa.

Con carácter previo al contraste del modelo planteado en la Figura 2, se ha procedido a verificar las propiedades psicométricas de las escalas. Concretamente, para los ítemes de personalidad se ha realizado un análisis factorial exploratorio (SPSS 16) a fin de identificar posibles dimensiones subyacentes. Este análisis revela la existencia de tres dimensiones con un autovalor superior a I (Cuadro 3). La primera dimensión, que hemos denominado sociabilidad, aúna a los ítemes relativos a la relación y compromiso que el emprendedor mantiene con otras personas; explica un 35, I\% de la varianza. La segunda dimensión-creatividad-recoge aquellos ítemes relativos a la creación de ideas, identificación y desarrollo de oportunidades empresariales y explica el । I,8\% de la varianza. Finalmente, la tercera dimensión comprende aspectos relativos a la importancia del éxito y la reputación para el individuo; explica un 9,9\% de la varianza y la hemos denominado imagen personal. Para el contraste del modelo planteado, por lo tanto, utilizaremos estas tres subdimensiones de la personalidad. Respecto al resto de variables multi-item, se ha comprobado la fiabilidad con el alpha de Cronbach y en todos los casos está por encima de 0,70, con la excepción de la imagen personal, en la que se queda en 0,69. La puntuación de todas las variables para los análisis posteriores se ha calculado como la media de los ítemes que las forman.

\section{Resultados}

Para la estimación del modelo empleamos un análisis path (AMOS 16)2. El valor de los coeficientes estandarizados significativos se presenta en la Figura 3. En primer lugar, cabe señalar que -como se esperaba- se confirma la existencia de un efecto directo de la conducta innovadora en el éxito empresarial $(\beta=0.08)$. Respecto a las variables de personalidad, únicamente la denominada creatividad tiene una influencia directa y positiva en la conducta innovadora $(\beta=0.24)$. Además este rasgo de la personalidad tiene una influencia directa sobre el éxito empresarial $(\beta=0.23)$. El efecto total de la creatividad en el éxito empresarial, suma del efecto directo $(0,24)$ e indirecto $(0,24 * 0,08)$, es de $\beta=0.26$. La sociabilidad no influye en la conducta innovadora, pero si tiene una relación negativa significativa con el éxito empresarial $(\beta=-0.1$ I). Por su parte, la experiencia laboral, al igual que la creatividad, tiene un efecto directo sobre la innovación $(\beta=0.1$ I) y sobre el éxito $(\beta=0.08)$. Como consecuencia de ello, el efecto total sobre el éxito es de $\beta=0.09$.

Cuadro 3

Análisis factorial de los ítemes de personalidad

\begin{tabular}{|c|c|c|c|}
\hline PERSONALIDAD & $\begin{array}{l}\text { FACTOR I } \\
\text { Sociabilidad }\end{array}$ & $\begin{array}{l}\text { FACTOR } 2 \\
\text { Creatividad }\end{array}$ & $\begin{array}{l}\text { FACTOR } 3 \\
\text { Imagen } \\
\text { personal }\end{array}$ \\
\hline $\begin{array}{l}\text { Tiene facilidad para generar nuevas ideas. } \\
\text { Tiene facilidad para empezar nuevas actividades. } \\
\text { Identifica oportunidades donde nadie las observa. } \\
\text { Si decide hacer algo, lo hará y nadie podrá detenerle. }\end{array}$ & & $\begin{array}{l}0,733 \\
0,807 \\
0,785 \\
0,592\end{array}$ & \\
\hline $\begin{array}{l}\text { Le gusta intercambiar opiniones e ideas con otras personas. } \\
\text { Tiene facilidad para relacionarse con la gente. } \\
\text { Procura que sus decisiones sean positivas sobre las personas. } \\
\text { Procura cumplir con los compromisos que asume. }\end{array}$ & $\begin{array}{l}0,725 \\
0,648 \\
0,734 \\
0,712\end{array}$ & & \\
\hline $\begin{array}{l}\text { Le gusta disfrutar y ser reconocido por sus éxitos personales. } \\
\text { Cree que el emprendedor de éxito tiene reconocimiento social. } \\
\text { En los actos públicos tiene cuidado con los aspectos de protocolo. } \\
\text { Le gusta preservar y mantener su reputación. }\end{array}$ & & & $\begin{array}{l}0,716 \\
0,787 \\
0,547 \\
0,600\end{array}$ \\
\hline $\begin{array}{r}\text { Autovalor } \\
\% \text { varianza explicada }\end{array}$ & $\begin{array}{c}4,33 \\
35,1 \%\end{array}$ & $\begin{array}{l}I, 4 \mathrm{I} \\
\mid \mathrm{I}, 8 \%\end{array}$ & $\begin{array}{l}1,19 \\
9,9 \%\end{array}$ \\
\hline
\end{tabular}

\footnotetext{
2 La determinación de los parámetros la realizamos por el método de la máxima verosimilitud. Este método asume que existe normalidad multivariable, asunción que comprobamos usando el test de Mardia. El gran valor de este estadístico indica la presencia de no normalidad. Para hacer frente a esta contingencia, utilizamos bootstrapping con la idea de confirmar la significación de los parámetros a partir del intervalo de confianza obtenido en torno al correspondiente estadístico.
} 
Pero, lo que realmente es el objetivo de este trabajo es constatar si, más allá del efecto atribuido a la personalidad y a la experiencia, existe una influencia de los programas de formación. De las tres dimensiones de la formación de los emprendedores consideradas -formación emprendedora propiamente dicha, formación especializada en áreas de la empresa y formación reglada- sólo la primera tiene un efecto directo positivo sobre la conducta innovadora $(\beta=0.16)$, ya que la formación reglada tiene un efecto negativo $(\beta=-0.09)$.

\section{Conclusiones}

El principal rasgo de la personalidad de un individuo, cuando de lo que se trata es de reforzar los resultados de la innovación, es la creatividad (Helfat y Raubitschek, 2003; Ward, 2004; Popadiuk y Choo, 2006). Es decir, los individuos con facilidad para generar nuevas ideas, empezar actividades, identificar oportunidades y desarrollarlas, están más satisfechos con su conducta innovadora que los que no están dotados de estas cualidades. Además, se ha mostrado que este rasgo de la personalidad tiene una influencia directa sobre el éxito no canalizada a través de la innovación. Por ejemplo, los individuos creativos pueden ser más eficientes desarrollando una política de comunicación original o gestionando la reducción de costes.
En este estudio, el efecto total de la creatividad en el éxito empresarial es más fuerte que el de cualquiera de las otras variables utilizadas en este estudio. La implicación que se deriva de esta conclusión es doble. Por un lado, las empresas deberían valorar muy positivamente este rasgo de la personalidad en su política de reclutamiento de personal y, por otro -aunque es obvio que se precisan ciertas condiciones innatas-, las instituciones de enseñanza tendrían que centrarse en estimular y potenciar este rasgo personal. Tal es la importancia concedida a esta característica del emprendedor que, en universidades de prestigio como la Harvard Business School, se realizan, periódicamente, congresos y seminarios en los que se analiza las formas de desarrollar este aspecto de la personalidad («Fourth Annual Research Conference on "Creativity, Entrepreneurship, and Organizations of the Futures)).

La sociabilidad no influye en la conducta innovadora, pero si tiene una relación negativa significativa con el éxito empresarial. Este resultado se puede interpretar como que el compromiso con las personas con las que se trabaja y el énfasis en que las decisiones satisfagan a tales personas puede mermar la rentabilidad. La conclusión, así expuesta, llevaría a cuestionar este rasgo de la personalidad como un elemento atractivo para el desarrollo de una actividad empresarial. Sin embargo, debe tenerse presente que sólo se está considerando una dimensión del

Figura 3

Relaciones significativas

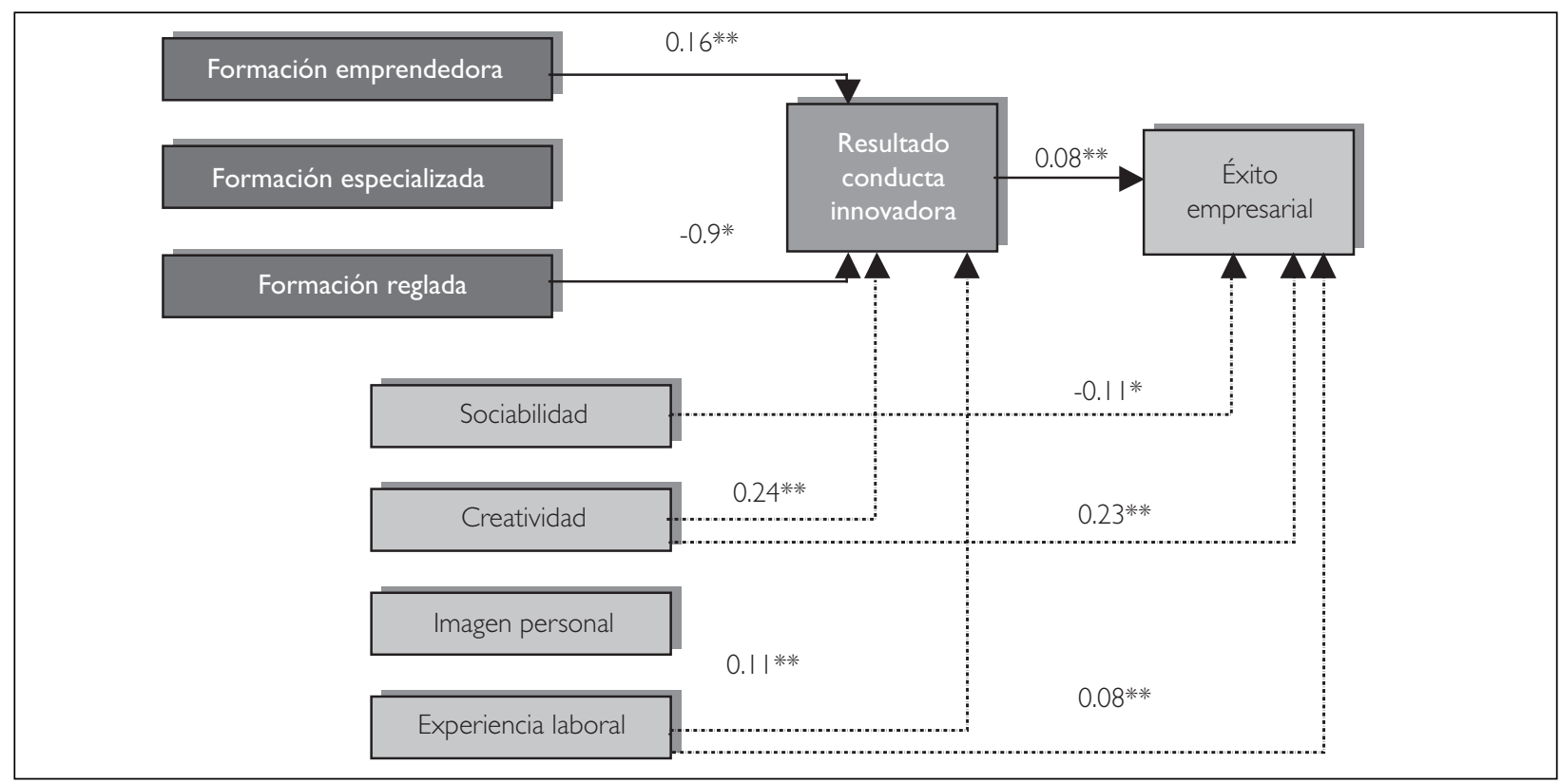

Nivel de significación (test de una cola): ** $p<0.01$, * $p<0.05$.

Estadísticos de ajuste: $X 2=8,54, \mathrm{DF}=7, \mathrm{p}=.29, \mathrm{NFI}=0.99, \mathrm{IFI}=1.00, \mathrm{RMSEA}=.02$. 
resultado (la rentabilidad); otros indicadores como el nivel de conflicto laboral o la satisfacción de los trabajadores, variables de las que cabe esperar una repercusión positiva en otras dimensiones del éxito y que pueden depender de esta dimensión de la personalidad, no han sido consideradas. Además, estadísticamente es fácil constatar que esta relación negativa existe sólo cuando en el análisis se incluye el efecto de la creatividad, como es nuestro caso. Si del análisis path realizado se excluye la personalidad, la relación se vuelve no significativa. Es decir, no existe ni una relación positiva ni negativa.

La experiencia laboral, al igual que la creatividad, tiene un efecto directo sobre la innovación y sobre el éxito. Por lo tanto, cabe recomendar como paso previo a la creación de una empresa y durante su funcionamiento enfrentarse al trabajo en diferentes áreas funcionales (Lazear, 2003). La consecuencia que de ello extraemos para el ámbito de la educación es que realizar prácticas en empresas, en la medida en que supone una primera experiencia profesional, puede ayudar a fomentar el espíritu innovador y el éxito empresarial, ya que dota al individuo de un conocimiento directo sobre el funcionamiento de la empresa y, por lo tanto, incrementa su sensación de control sobre este tipo de actividad.

A diferencia de estudios previos sobre la formación emprendedora en los que, tradicionalmente, se estudia el impacto de este tipo de formación sobre la intención de emprender o el comportamiento emprendedor (Florin et al., 2007; Souitaris et al., 2007), nuestro trabajo valora el efecto sobre la innovación y el éxito emprendedor. De las tres dimensiones de la formación de los emprendedores consideradas en este trabajo -formación emprendedora propiamente dicha, formación especializada en áreas de la empresa y formación reglada-, sólo la primera tiene un efecto directo positivo sobre la conducta innovadora. Esta relación indica que los individuos que se preocupan por el perfeccionamiento directivo y la capacitación emprendedora se muestran más satisfechos con la actividad de innovación que el resto. Además, de forma indirecta, a través de la relación entre innovación y éxito, la formación propiamente emprendedora contribuye a lograr mejores resultados empresariales, tal como se ha demostrado en investigaciones previas (Karlan y Valdivia, 2006). De forma notoria nuestros resultados conducen a recomendar a los centros de formación, e instituciones que los gestionan, que den preferencia a la formación específica en el ámbito del entrepreneurship, no a la formación generalista en aspectos em- presariales, cuando lo que quieren es reforzar el espíritu innovador de los emprendedores y, por esta vía, el éxito empresarial.

Haber alcanzado niveles de formación reglada superiores está relacionado con una cierta insatisfacción con la actividad de innovación. Esta insatisfacción puede derivarse tanto de la no realización de actividades de innovación como de una falta de logro de los resultados esperados. La segunda de las explicaciones la encontramos más factible, dado que nuestros datos revelan la existencia de una correlación positiva marginalmente significativa entre formación reglada y desarrollo de nuevos productos. Quizá, los individuos con mayor formación reglada, más seguros de sus conocimientos y menos conformistas, elevan su nivel de expectativas, y es la diferencia entre expectativas y resultados lo que genera insatisfacción. De cualquier forma, con independencia de este resultado, dada la relación antes comentada entre formación emprendedora y resultados, cabe recomendar la inclusión de este tipo de formación dentro de la formación reglada. Es patente su escasa importancia en los planes de estudio actuales (European Commission, 2008).

Particularmente relevante para cerrar este apartado de conclusiones e implicaciones es señalar que el nivel de formación de los individuos en aspectos puramente emprendedores es realmente bajo (véanse las medias del Cuadro 2). Todavía es importante el porcentaje de encuestados que afirman no haber realizado actividades de formación de este tipo. Además, cuando las han realizado, el número de horas ha sido bastante limitado. Por lo tanto, el margen de mejora es muy amplio.

Finalmente, cabe señalar que, dada la relación obtenida entre satisfacción con la innovación y éxito empresarial —completamente en línea con los argumentos expuestos por Holmstrom (1989)_, las variables que funcionan como antecedentes positivos de la innovación son, a su vez, variables que contribuyen al éxito empresarial. Esto es: la formación en aspectos emprendedores redunda en el éxito del proyecto empresarial.

\section{Limitaciones y extensiones de la investigación}

Las limitaciones que presenta nuestra investigación están relacionadas principalmente con la medición de las variables. Así, el comportamiento y resultado innovador y el éxito empresarial se han determina- 
do únicamente a partir de un ítem, lo que da una idea parcial de estos constructos, cuya naturaleza es mucho más compleja. Mejorar estas dos medidas, por ejemplo, valorar los diferentes tipos de innovación (de producto, de proceso, de mercado, etc.), no sólo la satisfacción que se obtiene, es una forma de avanzar en la línea de investigación que se sigue en este trabajo.

Por otra parte, se debe profundizar más en el conocimiento de algunas de las características de la formación. Por ejemplo, de qué calidad fue, con qué rigor se realizó, cuáles fueron los resultados obtenidos por el individuo, si se cursó a tiempo completo o parcial, cuántos años antes de emprender su aventura innovadora se efectuó, etc. Esta información nos ayudará a caracterizar la formación emprendedora más adecuada para el éxito. Hasta ahora hemos constatado la existencia de una relación positiva entre ambas variables, pero para diseñar programas altamente eficientes es pertinente conocer qué rasgos de la formación potencian más el éxito.

Finalmente, queremos señalar que aunque en este trabajo se postula que la ruta por la cual la formación influye en la conducta emprendedora es a través de la mejora de la actitud para emprender, un mayor conocimiento de los factores del entorno y un mayor control sobre ellos, esto realmente no se constata. Por lo tanto, es importante medir estás variables intermedias para confirmar realmente que la TCP es la base de la explicación de la influencia de la formación en la conducta innovadora.

\section{Bibliografía}

AGARWAL, R. y PRASAD, J. ( 1999): «Are individual differences germane to the acceptance of new information technologies?». Decision Sciences, 30 (2), 36 I-392.

AZJEN, I. ( 1987): «Attitudes, traits, and actions: Dispositional prediction of behavior in personality and social psychology»». En L. Berkowitz (Ed.), Advances in experimental social psychology (20, I-63). New York: Academic.

AJZEN, I. (2008): «Consumer attitudes and behavior». En C. P. Haugtvedt, P. M. Herr \& F. R. Cardes (Eds.), Handbook of Consumer Psychology (525- 548). New York: Lawrence Erlbaum Associates.

AJZEN, I. y MANSTEAD, A.S. (2007): «Changing health-related behaviors: An approach based on the theory of planned behavior». En K. van den Bos, M. Hewstone, J. de Wit, H. Schut \& M. Stroebe (Eds.), The scope of social psychology: Theory and applications (43-63). New York: Psychology Press.
AVLONITIS, G.J. y SALAVOU, H.E. (2007): «Entrepreneurial orientation of SMEs, product innovativeness, and performance». Journal of Business Research, 60 (5), 566 575.

BARON, R.A. (2004): «The cognitive perspective: a valuable tool for answering Entrepreneurship's basic «why» questions», Journal of Business Venturing, 19, 221 - 239.

BARON, R.M. y KENNY D.A. (1986): The moderator-mediator variable distinction in social psychological research: conceptual, strategic, and statistical consideration. Journal of Personality and Social Psychology, 5 | (6), | | 73- | I 82.

BAUM, J.R., LOCKE, E.A. y SMITH K.G. (200I): «A Multidimensional model of venture growth». Academy of Management Journal, 44 (2), 292-303.

BURKE, A., FITZROY, F.y M. NOLAN (2000): «When less is more: Distinguishing between entrepreneurial choice and performance». Oxford Bulletin of Economics and Statistics, 62(5), 565-587.

COMACCHIO,A., SCAPOLAN, A. y BONESSO, S. (2007): «Innovation, complementarities and performance in micro/small enterprises». International Journal of Entrepreneurship and Innovation Management, 7 (I), 5-28.

CROSS, B. y TRAVAGLIONE, A. (2003): «The untold story: is the entrepreneurship of the 2 I th century defined by emotional intelligence?». The International Journal of Organizational Analysis, I I (3), 22 I-228.

DJANKOV, S., MIGUEL, E., QIAN,Y., ROLAND, G. y ZHURAVSKAYA, E. (2005): «Who are Russia's entrepreneurs?», Journal of the European Economic Association, 3 (2-3), I- I I.

DYER, W.G. (1994): «Toward a theory of entrepreneurial careers». Entrepreneurship, Theory and Practice, 19 (2), 7-21.

EUROPEAN COMMISSION. ENTERPRISE AND INDUSTRY DIRECTORATE-GENERAL (2008): «Entrepreneurship in higher education, especially within nonbusiness studies», 69 .

FAYOLLE, A., GAILLY, B. y LASSAS-CLERC, N. (2006): «Assessing the impact of entrepreneurship education programmes: a new methodology»s. Journal of European Industrial Training, 30 (9), 701-720.

FISHBEIN, M. y AZJEN, I. ( 1975): Belief, Attitude, Intention, and Behavior:An Introduction to Theory and Research. Reading, MA: Addison-Wesley.

FLORIN, J., KARRI, R. y ROSSITER, N. (2007): «Fostering entrepreneurial drive in business education: An attitudinal approach». Journal of Management Education, 3 I ( I), 17-42.

HELFAT, C.E., RAUBITSCHEK, R.S. (2003): «Product sequencing: co-evolution of knowledge, capabilities, and products». En: Helfat, C.E. (Ed.), The SMS Blackwell Hand- 
book of Organizational Capabilities. Blackwell Publishers, Oxford, 193-340.

HISRICH, R. D., PETERS, M. P. y SHEPHERD D. A. (2005): «El empresario individual». Entrepreneurship: Emprendedores, capítulo 3, McGraw Hill, D. L., Madrid, 52-69.

HOLMSTROM, B. (1989): «Agency costs and innovation». Journal of Economic Behaviour and Organization, 12(3), 305-327.

HORNADAY, J.A. y ABOUD, J. ( 197 I): «Characteristics of successful entrepreneurs». Personnel Psychology, 24, I 4 I153.

HOWELL, J. M. (2005): «The right stuff: Identifying and developing effective champions of innovation», Academy of Management Executive, 19 (2), I08-1 19.

HYTTI, U. y KUOPUSJARVI, P. (2004): Evaluating and measuring entrepreneurship and enterprise education: Methods, tools and practices, Turku: Small Business Institute.

IAKOVLEVA, T. y KOLVEREID, L. (2005): «New firm performance: conceptual perspectives», Entrepreneurship Research in Europe: some key issues, chapter 4, Cheltenham: Edward Elgar, 80-101.

KARLAN, D.S. y VALDIVIA, M. (2006): «Teaching Entrepreneurship: Impact of Business Training on Microfinance Clients and Institutions». Yale University Economic Growth Center Discussion Paper no. 94I; Center for Global Development Working Paper no. 108.

KATZ, J.A. (2003): «The chronology and intellectual trajectory of American entrepreneurship education 1976 | 999»». Journal of Business Venturing, | 8, 283-230.

KRUEGER, N.F. y CARSRUD, A.L. (1993): «Entrepreneurial intentions: applying the theory of planned behavior». Entrepreneurship and Regional Development, 5, 315-330.

LAZEAR, E.P. (2003): «Entrepreneurship». IZA Discussion Paper, no. 760.

MACKINNON, D.P., KRULL J.L. y LOCKWOOD C.M. (2000): Equivalence of the mediation, confounding and suppression effect. Prevention Science, I (4), |73- I8I.

MAYER-SCHÖENBERGER,V. (2007): «Schumpeterian Policy Makers: Pro-Active Policies for Innovative Entrepreneurship». Hudson Institute Research Paper No. 07-04.
MESCON,T.S. y MONTANARI, J.R. ( 1981 ): «The Personalities of Independent and Franchise Entrepreneurs, An Empirical Analysis of Concepts», Academy of Management Conference, 4I3-4I7.

POPADIUK, S. y CHOO, C.W. (2006): «Innovation and knowledge creation: how are these concepts related?», International Journal of Information Management, 26, $302-312$.

ROBINSON, P.B., STIMPSON, D.V., HUERFNER, J.C. y HUNT, H.K. (199|): «An attitude approach to the prediction of entrepreneurship». Entrepreneurship Theory and Practice, I 5(4), I3-32.

SCHULZE, A. y HOEGL, M. (2008): «Organizational knowledge creation and the generation of new product ideas: A behavioral approach», Research Policy, 37 (10), 1742- 1750.

SCHUMPETER, J. A. (1934): The Theory of Economic Development, Cambridge, Mass.: Harvard University Press.

SHROUT P.E. y BOLGER, N. (2002): Mediation in experimental and nonexperimental studies: New procedures and recommendations. Psychological Methods, 7 (4), 422445.

SOUITARIS,V., ZERBINATI, S. y AL-LAHAM,A. (2007): «Do entrepreneurship programmes raise entrepreneurial intentions of science and engineering students? The effects of learning, inspiration and resources». Journal of Business Venturing, 22 (4), 566-59l.

UTSCH, A. y RAUCH, A. (2000): «Innovativeness and initiative as mediators between achievement orientation and venture performance». European Journal of Work and Organizational Psychology, 9 ( I), 45-62.

WANDOSELL, G. y GARCIA, A. (2004): «Motivaciones y obstáculos en la creación de empresas, efectos de la experiencia empresarial». Boletín Económico del ICE, 2819, | $1-17$.

WARD, T.B. (2004): «Cognition, creativity, and entrepreneurship». Journal of Business Venturing, 19, 173-188.

YONG, G. y HO, K.W. (2007): «Innovation, Imitation and Entrepreneurship». Singapore Economic Review, forthcoming.

\section{Nueva web de Dirección y Organización www.revistadyo.com}

